\title{
Using brain-computer-interface for robot arm control
}

\author{
Sebastian-Daniel Rosca ${ }^{1}$, and Monica Leba ${ }^{1, *}$ \\ ${ }^{1}$ University of Petroșani, Department of Computer and Electrical Engineering, 332006, Petroșani, \\ Romania
}

\begin{abstract}
In this paper, we analyze the principles of brain computer interface that convert the electrical activity of the human brain into commands for a computer of any embedded device. For this reason, we present the existing devices and applications from the area of braincomputer interfaces with advantages and disadvantages. Further, we propose a solution for brain control of a robotic arm. We develop the model and simulate the entire system functioning, both the robotic arm control and the brain signals processing. The final purpose of our research is to achieve a brain-computer system that controls a robotic arm that can replace a human arm.
\end{abstract}

\section{Introduction}

Over the time, people have fantasized about the ability to communicate and interact with the machine by simply thinking or creating devices that read thoughts. These ideas have captured people's imagination in the form of ancient myths and science fiction stories.

In recent years, control devices „,by the power of the mind” is a very controversial topic, but highly researched, both in the last generation gadget, such as smart phones, laptops and tablets and even televisions intelligent, but also in medicine, to be used by people with disabilities for which these technologies could be the only way of communication with the external environment. Today, brain-machine interfacing (BMI) development [braincomputer interfacing or (BCI)] has a rapid growth of research because it provides a unique way of communication between a human and a machine (or device) without any neuromuscular intervention [1].

Brain-computer interfaces are often used to help paralyzed people or with motor functions diminished by connecting to a robotic arm; it's important that these people should not be fixed in one place, as it happens when they are connected to the interface braincomputer through a cable, but they can move around the room - thus defending the interest for a wireless connection. The basic idea of a BCI system is to measure electrical activity, magnetic or any other physical manifestations of brain activity and convert them into commands for a computer or any other device (wheelchair, neuroprosthesis). A BMI is a system based on digital signal processing tools and machine learning how to identify and predict the user's cognitive state from corresponding brain signal [1].

*Corresponding author: monicaleba@upet.ro 
The brain signals are recorded either invasively or noninvasively [1]. Noninvasive means are widely used by most BMI/BCI researchers for their simplicity in user interface, although invasive means provides better performance in terms of accuracy and precision. The most widely used noninvasive recording technique is electroencephalography (EEG where the signals are recorded by electrodes placed on the scalp) because it is inexpensive, portable, easily available and has high temporal resolution [1].

\section{Applications}

Neurospeller application ensure a way for communication for people that suffer from severe communication disabilities gained from neurological diseases and offers a new mode for human interaction to improve their life [2]. BCI applications for communication purposes are based on a virtual keyboard [3] that is shown on the display that offers the user possibility to select a letter from the alphabet by means of a Brain-Computer Interface (BCI) [4].

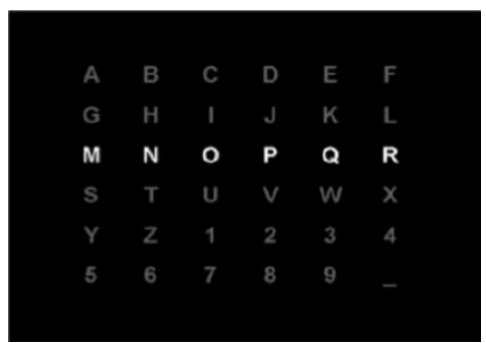

Fig. 1. User display in P300 Speller [3].

Wheelchair Control with Mental Tasks could be represent a real help for person that suffer from hemiplegia because they don't have a full control of their body and they cannot use a joystick of an electric wheelchair [5]. This technology offers for these person a chance to obtain independence and don't have any more to request help from a companion to push their wheelchair [6].

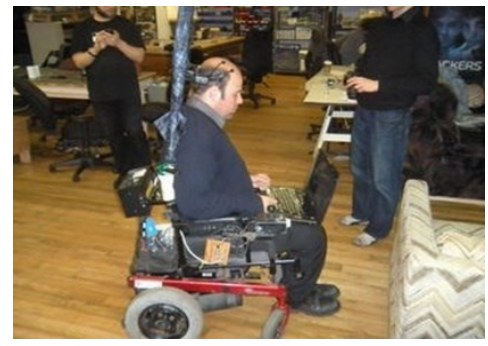

Fig. 2. BCI - Brain-Controlled-Wheelchair [5].

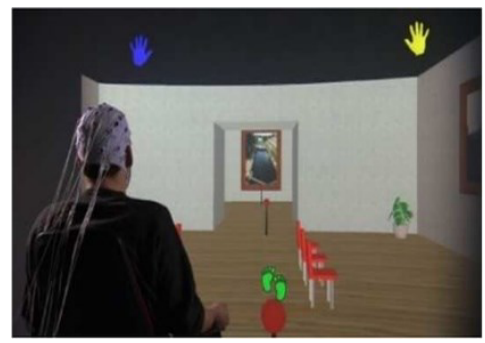

Fig. 3. BCI-based navigation in a complex VE [9]. 
Brain-controlled video gaming technology can be used both for medical and nonmedical purposes [7]. As possible purposes can be: to allow the persons with severe mobility deficiency (e.g. spinal cord injuries) that cannot use a keyboard, a mouse, or a joystick to play video-games; to recover the lost functions result from an disease or a trauma at the level of brain (e.g. stroke or neurodegenerative diseases); for relaxation and entertainment to bring experience to a new level [8].

Brain-controlled Robotic Prosthetic Limboffers a solution for people who have suffered limb amputations resulted in the development of Brain-Computer Interface for controlling robotic limb prosthesis through EEG signals that offer a better solution than the existing ones (e.g. control of robotic human prosthesis by electromyographic sensors placed on the muscles of healthy limb to replicate the movements) and can be helpful for completely paralyzed peoples [10].

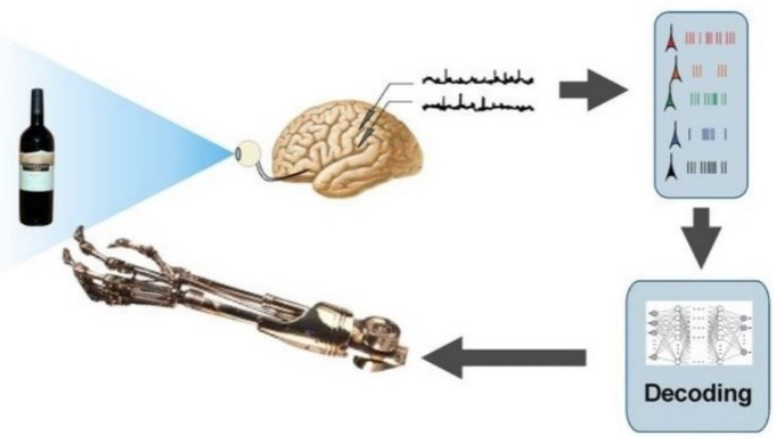

Fig. 4. Diagram of a neural prosthetic system [11].

\section{Software}

BioSig is an open source software library developed by Graz University of Technology for biomedical signal processing. BioSig provides open source software tools for many different areas applications: neuroinformatics, brain-computer interfaces, neurophysiology, psychology, cardiovascular systems and sleep research [12].

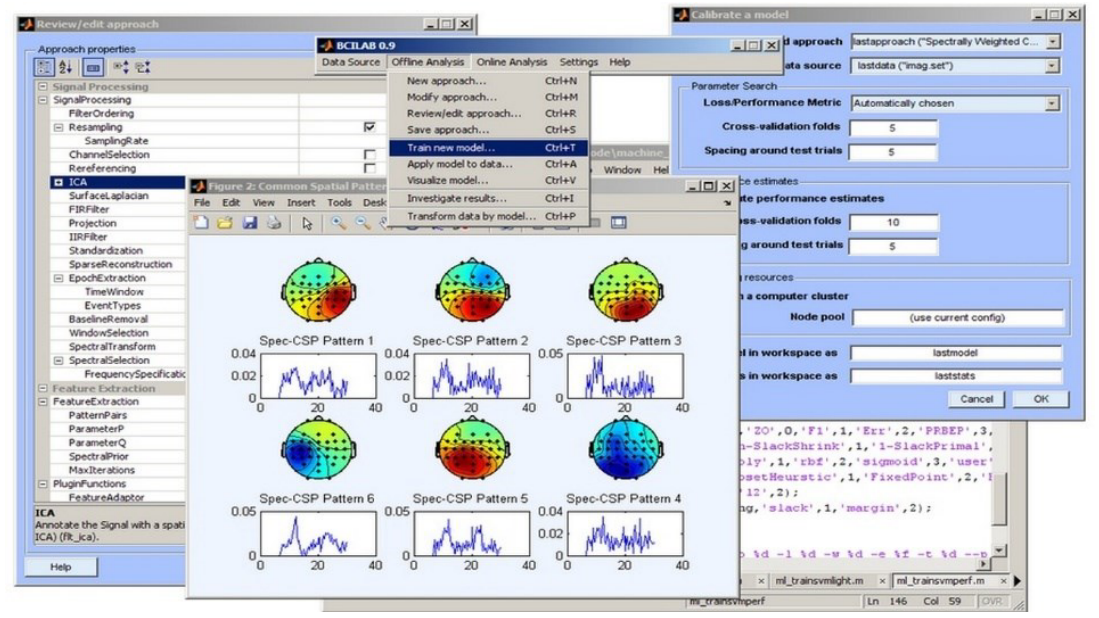

Fig. 5. BCILAB Sample GUI. 
OpenViBE is developed by INRIA and is a software platform used to designing, testing and using brain-computer interfaces. OpenViBE is a free open source software for real-time neurosciences (real-time processing of brain signals) because it can be used to acquire, filter, process, classify and visualize brain signals in real time. Some of OpenViBE application fields are: medical (assistance to disabled people, real-time biofeedback, neurofeedback, real-time diagnosis), multimedia (virtual reality, video games), robotics and all other application fields related to brain-computer interfaces and real-time neurosciences.

BCILAB is a MATLAB toolbox and EEGLAB plug-in for the design, prototyping, testing, experimentation with, and evaluation of Brain-Computer Interfaces (BCIs) and other systems in the same computational framework. The toolbox has been developed by $\mathrm{C}$. Kothe at the Swartz Center, inspired by the preceding PhyPA BCI toolbox created by C. Kothe and T. Zander at the Chair for Human-Machine Systems, Berlin Institute of Technology [13].

\section{Brain controlled robotic arm}

The system developed in this paper, which is still in research and needs improvements, aims to replace lost functions due to amputations for the human arm. The system includes a robot arm with five fingers and BCI headset with five channels.

In accordance with the real model of a human arm that provides seven degrees of freedom, the proposed robot arm provides 7 degrees of freedom for arm and 16 degrees of freedom for hand [14].

The robot allows seven types of movement along the $\mathrm{x}, \mathrm{y}$, and $\mathrm{z}$ axes, as follows: 3 degrees of freedom for the shoulder joint (movement right-left, up-down and forwardback); 2 degrees of freedom, 1 for the elbow joint (movement forward-back/flexionextension) and 1 for forearm (pronation/supination); 2 degrees of freedom for the wrist (rotation between the shoulder-elbow and elbow-wrist parts).

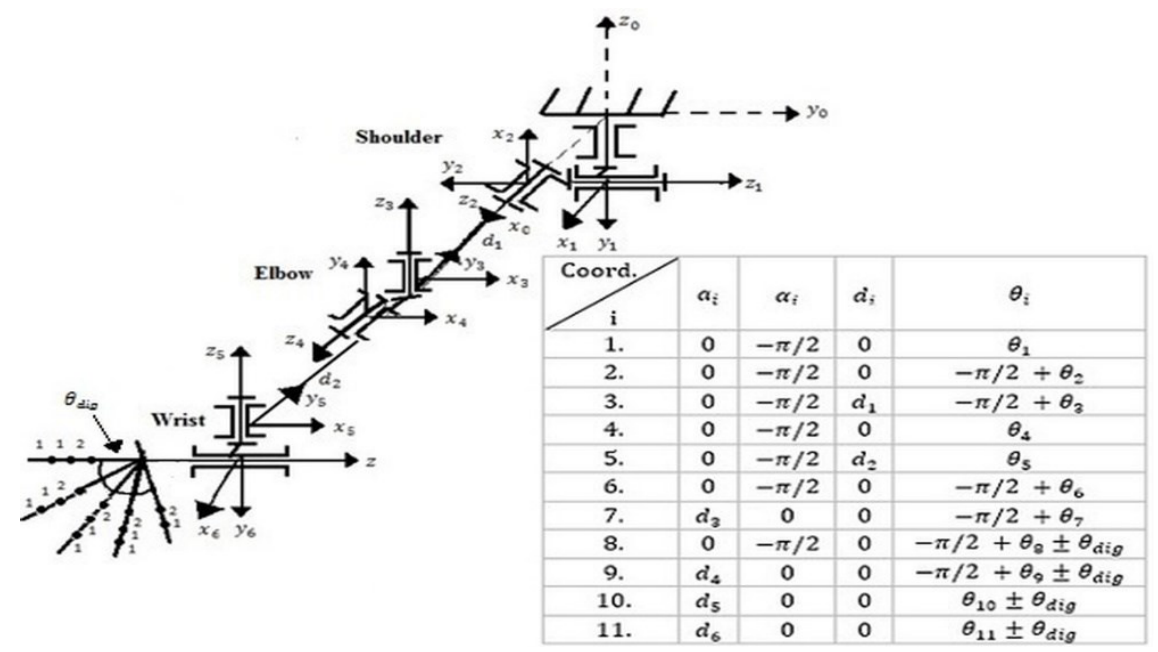

Fig. 6. Robot arm outline.

We apply the Denavit-Hartenberg formalism to determine the direct kinematic model of the robot arm in order to model and simulate the movement in MatLab-Simulink.

Using the parameters identified in the Denavit-Hartenberg table from figure 6, we determine the movement matrix for each finger, based on the formula: 


$$
T_{i-1, i}=\left[\begin{array}{cccc}
\cos \theta_{i} & -\sin \theta_{i} \cos \alpha_{i} & \sin \theta_{i} \sin \alpha_{i} & a_{i} \cos \theta_{i} \\
\sin \theta_{i} & \cos \theta_{i} \cos \alpha_{i} & -\cos \theta_{i} \sin \alpha_{i} & a_{i} \sin \theta_{i} \\
0 & \sin \alpha_{i} & \cos \alpha_{i} & d_{i} \\
0 & 0 & 0 & 1
\end{array}\right]
$$

The mathematical model for the robot arm was next simulated in MatLab-Simulink. In figure 7 there is presented the shoulder joint formed up by three revolute simple joints linked by bodies of 0 lengths. We adopted this solution in order to point out the structure of the shoulder joint and to be able to accurately control the three revolution movements for this element. All the other joints are similar to this.

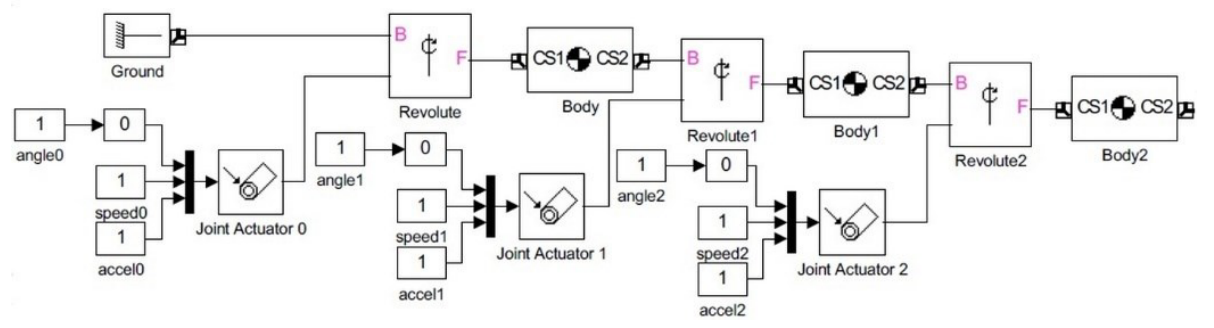

Fig. 7. MatLab-Simulink model of the shoulder part of the robotic arm.

The robotic hand is equipped with five high-end servomotors for advanced robotic applications that can operate the 16 joints of the fingers, either individual or in the same time for the gripping operations.

Entire parts of the robotic arm were projected in computer aided design software and were printed from Polylactic acid plastic (PLA) using a 3D printer. The BCI headset that was used in our research is Emotiv Insight.

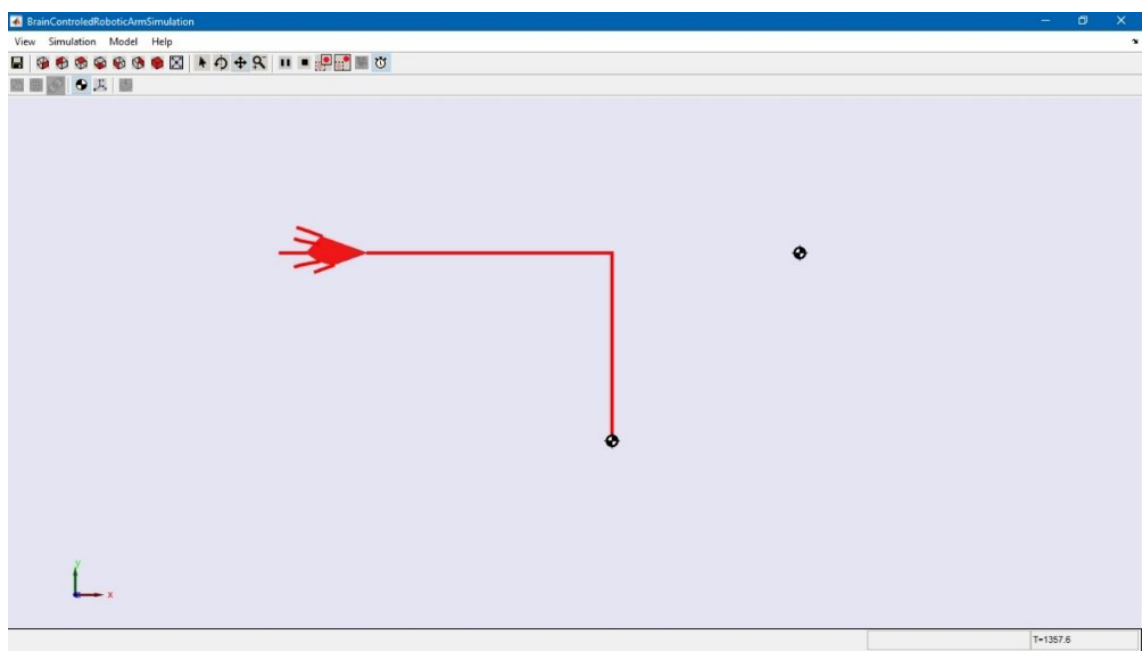

Fig. 8. Simulation of robotic arm $\left(90^{\circ}\right)$.

The Emotiv Xavier Control Panel interface that we use for control the human robotic arm through mental commands is a revolutionary interface for human-computer interaction. The interface allows 13 types of movement of a virtual object. After that movements are training, the Emotiv Insight headset create a pattern based on EEG principle and signals are convert into a command that can be understood by a computer.

We proposed to use 4 mental commands for control the human robotic arm in four complex movements. The mental commands chosen are the follows: 
- lift mental command (fig. 9) for up the shoulder along the $z_{1}$ axis $\left(0^{\circ}-90^{\circ}\right)$ and flexion the ellbow along $y_{3}$ axis $\left(0^{\circ}-90^{\circ}\right)($ fig. 8,9$)$

- drop mental command for down the shoulder joint along the $z_{1}$ axis $\left(90^{\circ}-0^{\circ}\right)$ and extension the ellbow along $y_{3}$ axis $\left(90^{\circ}-0^{\circ}\right)$

- rotate right mental command for close the human robotic hand

- rotate left mental command for open the human robotic hand

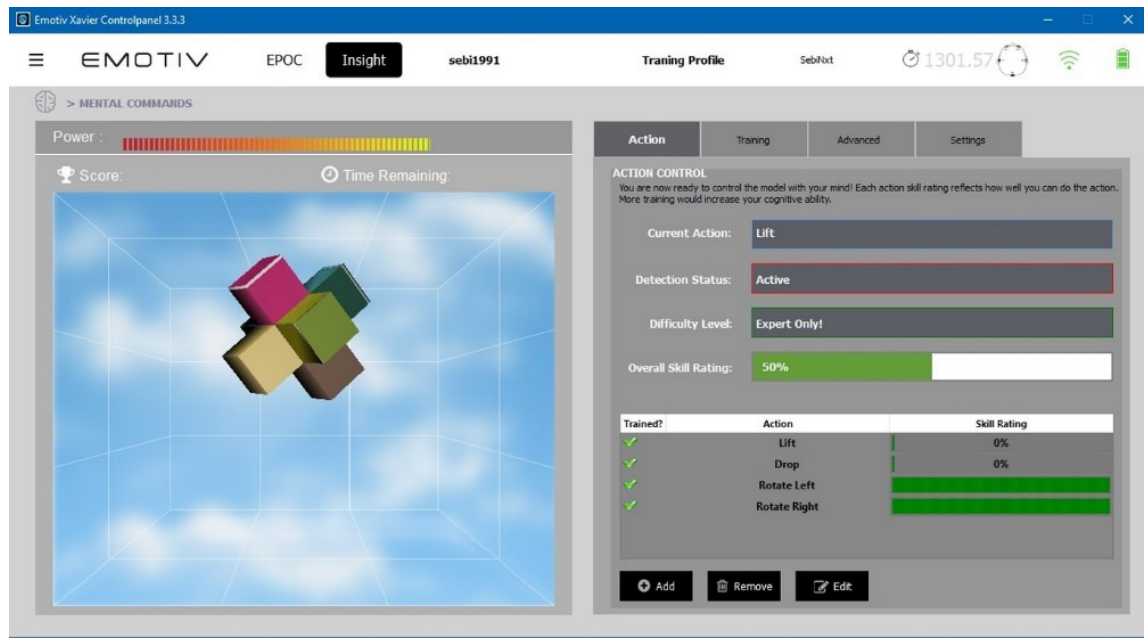

Fig. 9. Interface Emotiv Xavier Control Panel for lift mental command.

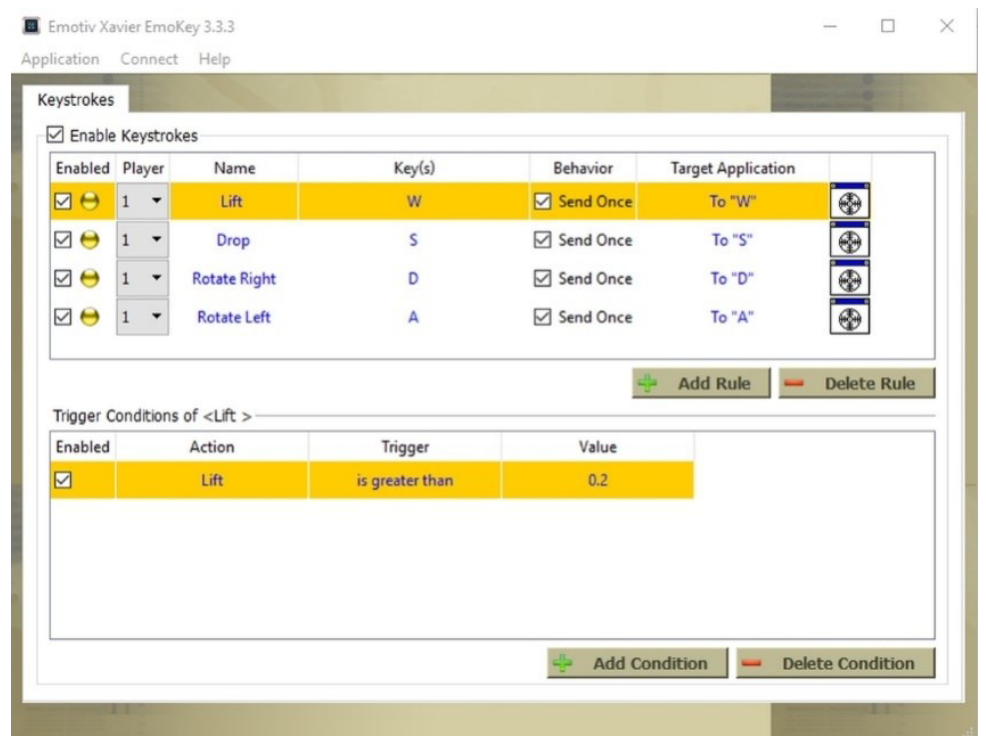

Fig. 10. Emotiv XavierEmoKey user interface.

In figure 8 is presented the simulation results for a lift command.

In figure 9 is presented the corresponding brain computer interface. As can be seen the mental command for arm lift resulted from the trainning, based on EEG and focus signals.

Emotiv XavierEmoKey user interface (fig.10) converts the results obtain after the measurements of mental command in predefined key sequences based on logical rules Mapping EmoKey defined by the user. We assigned a set of rules for convert the mental commands in keystroke event. 


\section{Conclusions}

Brain-computer interface is a revolutionary technology that opens a path from a new era of human computer interaction both by the fact that it addresses a large number of target groups but also that it can be a real help for making daily activities much easier.

In this research we propose a robot arm that has all the joints' movements specific to the human arm. We developed the Denavit-Hartenberg model for this robot arm and simulated it in MatLab-Simulink in order to validate its use.

Then, there was developed a simple brain control interface based on a commercial BCI headset in order to prove the possibility to brain control such a robot arm using 4 mental commands, 2 of them for the shoulder movement and 2 of them for the hand.

We propose as further research to implement within the brain-controlled robotic arm a series of pressure sensors for each finger to control the objects grip force. Also, we propose to develop a method to real-time adapt the brain-controlled robotic arm movements with the goal of improving response time and performing natural movements.

\section{References}

1. S. Bhattacharyya, S. Shimoda, M. Hayashibe, IEEE Transactions on Systems, Man and Cybernetics: Systems, 46(7), 957 (2016).

2. L. F. Nicolas-Alonso, J. Gomez-Gil, Sensors 12(2), 1249 (2012), (Available at: http://doi.org/10.3390/s120201211)

3. M. Thulasidas, C. Guan, J. Wu, IEEE Transactions on Neural Systems and Rehabilitation Engineering, 14(1), 24 (2006)

4. R. Fazel-Rezai, B. Z. Allison, C. Guger, E. W. Sellers, S. C. Kleih, A. Kübler, Frontiers in Neuroengineering, 5(14), (Available at: http://doi.org/10.3389/fneng.2012.00014, 2012)

5. $* * * h t t p: / / w w w . i n s t r u c t a b l e s . c o m / i d / B r a i n-C o n t r o l l e d-W h e e l c h a i r /$, Accesed 8.03.2017

6. T. Kaufmann, A. Herweg, A. Kübler, Journal of Neuro Engineering and Rehabilitation, 11(7), 15 (Available at:http://doi.org/10.1186/1743-0003-11-7, 2014)

7. D. Coyle, J.Principe, F. Lotte, A. Nijholt, IEEE Transactions on Computational Intelligence and $\mathrm{AI}$ in games, 5(2), 77 (2013)

8. C. G. Lim, T. S. Lee, C. Guan, D. S. S. Fung, Y. Zhao, et al, PloS one, 7(10), e46692., (Available at: doi:10.1371/journal.pone, 2012)

9. F. Lotte, Proceedings of the 6th International Conference on Foundations of Digital Games. ACM Jun 2011, Bordeaux, France, 325, (2011).

10. C. Guger, W. Harkam, C. Hertnaes, G. Pfurtscheller, Proc. AAATE $5^{\text {th }}$ european conference for the advancement of assistive technology, 3, (1999)

11. C. Pedreira, J. Martinez, R. Q. Quiroga, (Available at: https://www2.le.ac.uk/departments/engineering/research/bioengineering/neuroengineeringlab/Publications/PID924857.pdf, 2009)

12. *** http://biosig.sourceforge.net/, Accesed 1.05.2017

13. ***https://sccn.ucsd.edu/wiki/BCILAB, Accesed 11.02.2017

14. A. Ionica, M. Leba, Procedia Economics and Finances 23, 671 (2015) 